\title{
Current clinical practice: differential management of uveal melanoma in the era of molecular tumor analyses
}

\author{
This article was published in the following Dove Press journal: \\ Clinical Ophthalmology \\ 3 December 2014 \\ Number of times this article has been viewed
}

\section{Thomas M Aaberg Jr' \\ Robert W Cook ${ }^{2}$ \\ Kristen Oelschlager ${ }^{2}$ \\ Derek Maetzold ${ }^{2}$ \\ P Kumar Rao ${ }^{3}$ \\ John O Mason III}

'Michigan State University Medical School and Retina Specialists of Michigan, Grand Rapids, MI, ${ }^{2}$ Castle Biosciences, Friendswood, TX, ${ }^{3}$ Washington University School of Medicine in St Louis, St Louis, MO, ${ }^{4}$ Retina Consultants of Alabama and University of Alabama Birmingham, Birmingham, AL, USA
Correspondence: Thomas M Aaberg Jr Michigan State University Medical School and Retina Specialists of Michigan, 2757 Leonard St NE, Grand Rapids, MI 49525, USA

Tel + I 6169542020

$\mathrm{Fax}+\mathrm{I} 6169490408$

Email thomasaaberg@comcast.net
Objective: Assess current clinical practices for uveal melanoma (UM) and the impact of molecular prognostic testing on treatment decisions.

Design: Cross-sectional survey and sequential medical records review.

Participants: Ophthalmologists who treat UM.

Methods: (A) Medical records review of all Medicare beneficiaries tested by UM gene expression profile in 2012, conducted under an institutional review board-approved protocol. (B) 109 ophthalmologists specializing in the treatment of UM were invited to participate in 24-question survey in 2012; 72 were invited to participate in a 23-question survey in 2014.

Main outcome measures: Responses analyzed by descriptive statistics, frequency analyses (percentages, Tukey, histograms), and Fisher's exact test. Descriptive presentation of essay answers.

Results: The review of Medicare medical records included 191 evaluable patients, 88 (46\%) with documented medical treatment actions or institutional policies related to surveillance plans. Of these 88, all gene expression profiling (GEP) Class 1 UM patients were treated with low-intensity surveillance. All GEP Class 2 UM patients were treated with high-intensity surveillance $(P<0.0001$ versus Class 1$)$. There were $36(19 \%)$ with information concerning referrals after initial diagnosis. Of these 36, all 23 Class 2 patients were referred to medical oncology; however, none of the 13 Class 1 patients were referred $(P<0.0001$ versus Class 1$)$. Only Class 2 patients were recommended for adjunctive treatment regimens. 2012 survey: 50 respondents with an annual median of 35 new UM patients. The majority of respondents $(82 \%)$ performed molecular analysis of UM tumors after fine needle biopsy (FNAB); median: 15 FNAB per year; 2014 survey: 35 respondents with an annual median of 30 new UM patients. The majority offered molecular analyses of UM tumor samples to most patients. Patients with low metastatic risk (disomy 3 or GEP Class 1 ) were generally assigned to less frequent (every 6 or 12 months) and less intensive clinical visits. Patients with high metastatic risk (monosomy 3 or GEP Class 2) were assigned to more frequent surveillance with hepatic imaging and liver function testing every 3-6 months. High-risk patients were considered more suitable for adjuvant treatment protocols.

Conclusion: The majority of ophthalmologists treating UM have adopted molecular diagnostic tests for the purpose of designing risk-appropriate treatment strategies.

Keywords: uveal melanoma, gene expression profiling (GEP), Medicare, molecular diagnostic test

\section{Introduction}

The most common primary intraocular cancer in the United States is uveal melanoma (UM), the second most frequent subcategory of melanoma. ${ }^{1}$ New UM cases occur at 
a rate of $\sim 4.3$ per million per year; UM has the distinction of being one of the few clinically-diagnosed malignancies. ${ }^{1-5}$ In addition, tumor tissue is rarely archived, because the majority of UM patients receive eye-sparing treatment of the primary tumor. Unfortunately, although less than $4 \%$ of patients have detectable metastatic disease at the time of initial diagnosis, $\sim 50 \%$ will eventually manifest distant tumors, primarily in the liver. Traditional staging methods that use clinical and histologic prognostic factors, such as the American Joint Committee on Cancer (AJCC) TNM system, can be used to stratify patients into general risk categories, but they do not provide sufficient predictive accuracy to be used for patient care. ${ }^{6}$ Based on AJCC and National Comprehensive Cancer Network $(\mathrm{NCCN})^{7}$ cancer management guidelines, a $50 \%$ risk of metastasis (or recurrence) generally correlates with Stage III disease, the closest example being cutaneous melanoma, and stage III disease is uniformly treated with high-intensity imaging and, when available, adjuvant chemotherapy, targeted therapy, or radiotherapy. Given the poor accuracy of the TNM staging system for UM, the management of UM patients has been historically variable, owing to the lack of clearly defined treatment guidelines. As a consequence, management of all patients as high-risk for tumor metastasis in some clinical practices may result in overmanagement of patients who were actually low-risk. Conversely, in other clinical practices, high-risk patients may be relatively undermanaged.

Loss of chromosome 3 is one of the key early cytogenetic alterations associated with more aggressive $\mathrm{UM},{ }^{8}$ and monosomy of chromosome 3 in as little as $6 \%$ of tumor cells significantly increases the risk of UM metastasis. ${ }^{9}$ However, intratumoral heterogeneity for monosomy 3 is a frequent occurrence that complicates accurate detection and is understandable, given that the majority of tumor specimens are obtained from a single pass fine-needle aspiration biopsy (FNAB). ${ }^{10-12}$ This FNAB approach is further complicated by the need for relatively large tumor samples, in order to perform the most common chromosomal detection methods, such as fluorescent in situ hybridization (FISH). As a result, technical failure in FNAB specimens has been reported in as many as $50 \%$ of cases. ${ }^{13-15}$

More recently, gene expression profiling (GEP) of UM has gained diagnostic acceptance among ocular oncologists..$^{3,4,16}$ GEP takes a "snapshot" of the tumor microenvironment that can be used to predict the metastatic potential of the tumor. Because tumor sample requirements are generally lower for GEP assay, it has a lower technical failure rate than chromosomal assays. ${ }^{17}$ GEP has been reported in multicenter studies to have superior predictive results versus clinical, pathological, or chromosomal analyses. ${ }^{3,4,8,16}$

Molecular analysis of UM requires FNAB samples. However, few reports exist in the medical literature documenting the specific FNAB practices used by ocular oncologists in UM patients. Even though molecular testing has been concluded to be a clinically significant prognostic factor, recommended by the AJCC, ${ }^{18}$ there are few data documenting the current frequency and uses of molecular tests in the UM patient population. The availability of validated, accurate prognostic information may impact the selection of a management or treatment plan, including surveillance, referral, and therapy initiation within the clinic or clinical trial environment that matches metastatic risk. The intent of this investigation is to assess the current clinical practices for UM and the impact of molecular prognostic testing on treatment decisions through (1) focused surveys among ophthalomologists who diagnose and treat UM patients, and (2) a sequential review of limited medical records and known documented policies for all Medicare beneficiaries whose GEP tests were successfully performed and records were available in 2012.

\section{Methods \\ Medicare medical records review}

A retrospective, treatment decision impact analysis of the medical records of patients for whom UM GEP testing was ordered and processed for routine clinical use in 2012 was performed. These medical records were acquired by Castle Biosciences, Inc., (Friendswood, TX) to fulfill Medicare requirements for medical record submission during insurance claims submissions and appeals. The medical chart records were limited to the timeframe, including diagnosis of UM through approximately 12 weeks following primary eye tumor treatment. The Medicare patients had been treated by one of 37 ophthalmologists in the US. Only data related to basic demographics, UM tumor pathology and diagnosis, clinical surveillance practices, and institutional treatment policies were extracted from the records. Documented medical policy statements pertaining to use of prognostic testing results were also included. This study was approved by the Western Institutional Review Board, Olympia, WA. The data were analyzed by descriptive statistics. Fisher's exact test was used to analyze the data for GEP Class versus 1) surveillance intensity, 2) oncology referral rates, and 3) adjuvant treatment.

For both the medical records review and the clinician survey, high-intensity surveillance of UM patients was 
defined as clinical visits every 3-6 months, liver function tests every 3-6 months, and liver imaging/systemic evaluation (eg, computed tomography [CT], ultrasound, magnetic resonance imaging [MRI]) every 3-6 months. Low-intensity surveillance of UM patients was defined as clinical visits every 6-12 months and liver function tests, with or without some type of hepatic imaging, once a year.

\section{Surveys of clinicians}

Two surveys were performed, one in 2012 and the other in 2013/14. In 2012, ocular oncologists (ophthalmologists with subspecialty training in ocular oncology via retina, ophthalmic pathology, or ocular oncology fellowships) treating UM in North America, South America, and Europe were invited to participate anonymously in a 24-question survey that explored the use of FNAB and molecular diagnostic tests in their clinical management of UM cases. This survey was conducted in response to an invitation from the American Association of Ophthalmologists to the lead author (Thomas M Aaberg Jr) to give a plenary talk on "Practical Approaches to Genetic Diagnosis for Ocular Melanoma" at their 2012 Retina Subspecialty Day meeting in Chicago, IL.

At the end of 2013/beginning of 2014, ocular oncologists treating UM in the US were invited to participate anonymously in a 23-question survey that explored the use of radiotherapy and molecular diagnostic testing of UM tumor biopsies in current clinical practice. The follow-up study was conducted to address topics not covered in the first survey, to investigate whether or not any changing trends in patient care could be detected, and to attempt to expand the respondent pool. The invitees included all known ocular specialists in practice at the time of each survey. The list of potential survey ocular oncologists was developed by referring to several ophthalmology association membership listings and through personal knowledge of the field by the authors, based on practice patterns of clinicians who diagnosed and managed UM patients. An online survey tool was used to capture responses. The survey questions and data analyses were developed by the authors. Survey responses were analyzed by descriptive statistics and frequency analyses (percentages, Tukey, histograms), with descriptive presentation of essay answers.

\section{Results}

\section{Medicare medical records review}

There were 191 evaluable Medicare medical records (of 195 total beneficiaries tested) for UM patients treated by
37 diagnosing physicians (Figure 1). The evaluable patient cohort was $57 \%$ male with a mean age of $72 \pm 8$ years $( \pm$ standard deviation). Fifty-eight percent of the patients were GEP Class 1 and 42\% were GEP Class 2. Of these cohorts, $91 \%$ of the Class 1 and $84 \%$ of the Class 2 tumor samples were comprised of FNAB, with the remainder from formalinfixed, paraffin-embedded enucleation tissue.

\section{Surveys of clinicians: general practices}

Fifty out of the 109 queried ocular oncologists participated in the 2012 survey designed to assess the prevalence of FNAB and molecular diagnostic tests for managing the treatment of UM patients ( $46 \%$ response rate). The median annual caseload of new UM patients for each ocular oncologist was 35 (mean \pm standard error of the mean [SEM]: 56 \pm 11 ) (Figure 2A). The majority ( $82 \%$ ) performed some type of cellular and/or molecular analysis of UM cases, requiring the use of tumor tissue FNAB. Eighty-seven percent of respondents (32 out of 37) analyzed all biopsy tumor samples from both FNAB and enucleated eyes, while the remainder analyzed only enucleated eyes. Safety concerns relating to tumor location or other features rendered a median of $10 \%$ of the UM patients ineligible for a biopsy procedure across the respondents' clinical practices.

Thirty-five out of the 72 queried ocular specialists participated in the 2014 survey (49\% response rate). Twelve (34\%) had not participated in the 2012 survey, seven (20\%) were unsure, and 16 (46\%) had previously participated in the 2012 survey. Of the respondents, $49 \%$ were based at a university, $46 \%$ were based in private practice, and two (5\%) were based at both. The majority (66\%) were the only physician in their practice group to treat UM, with $29 \%$ indicating that one other colleague in their practice group also treated UM, and only two respondents indicating that two or more other colleagues treated UM. The median annual caseload of new UM patients for each ocular oncologist was 30 (mean \pm SEM: $40 \pm 5$ ) (Figure 2B). GEP was offered to patients by $88 \%$ of respondents (30 out of 34 ), while $24 \%$ offered chromosome three analysis (CHR3) (8 out of 34).

\section{Surveys of clinicians: surgical techniques and radiotherapy}

As illustrated in Figure 2A, respondents in the 2012 survey performed a median of $15 \mathrm{UM}$ biopsies per year (mean: 27 \pm 7 ). Almost all (94\%) were conducted at the time of plaque placement, enucleation, or clip placement. Most respondents biopsied only one site (69\%), while $20 \%$ biopsied two sites. Few biopsied more than two sites (11\%). 


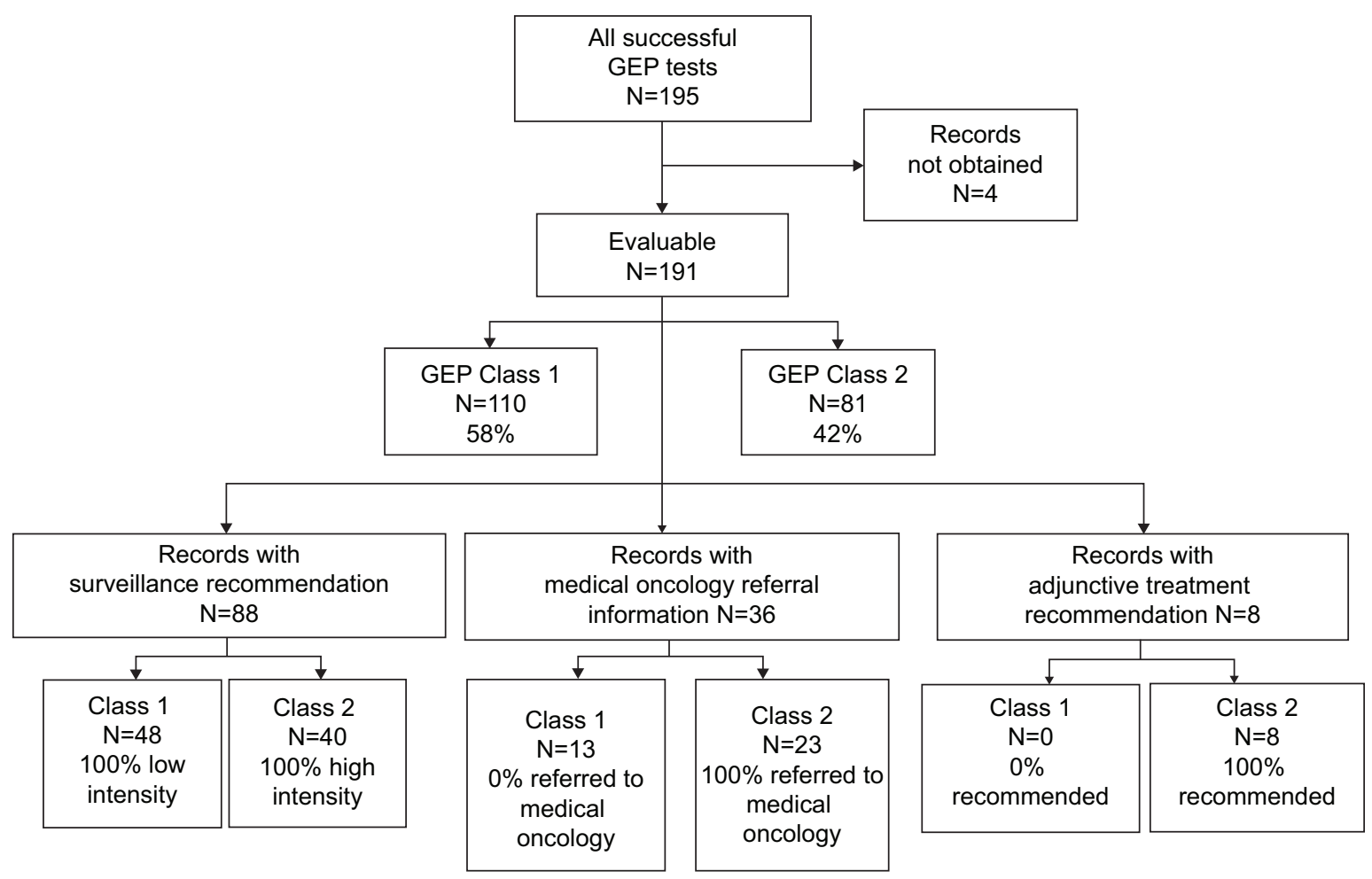

Figure I Summary of Medicare medical records review.

Notes: There were 191 evaluable Medicare medical records for UM patients treated by 37 diagnosing physicians. High-intensity surveillance of UM patients was defined as clinical visits every 3-6 months, liver function tests every 3-6 months, and liver imaging/systemic evaluation (eg, CT, ultrasound, MRI) every 3-6 months. Low-intensity surveillance of UM patients was defined as clinical visits every 6-12 months and liver function tests, with some type of hepatic imaging, at least once a year.

Abbreviations: UM, uveal melanoma; CT, computed tomography; MRI, magnetic resonance imaging; GEP, gene expression profiling.

The majority of respondents in the 2012 survey used a 25 (57\%) or 27 (43\%) gauge needle for the FNAB, and $77 \%$ used flexible tubing between the needle hub and a ten cc syringe, to stabilize the needle. Most respondents (74\%) did not use a vitrector for the biopsy procedure, although $9 \%$ used one for $50 \%$ or more of their surgeries. A median of $80 \%$ of biopsy procedures were performed transscleral (mean \pm SEM: $70 \pm 5$ ) and a median of $20 \%$ were performed transvitreal (mean \pm SEM: $30 \pm 5$ ). Choice of approach was generally determined by tumor location and depth, although several respondents indicated
A

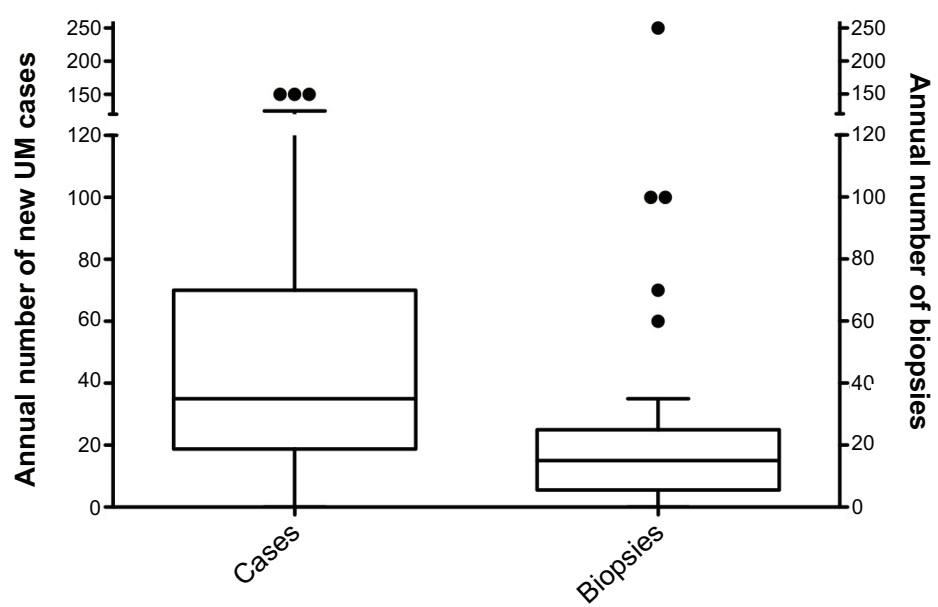

B

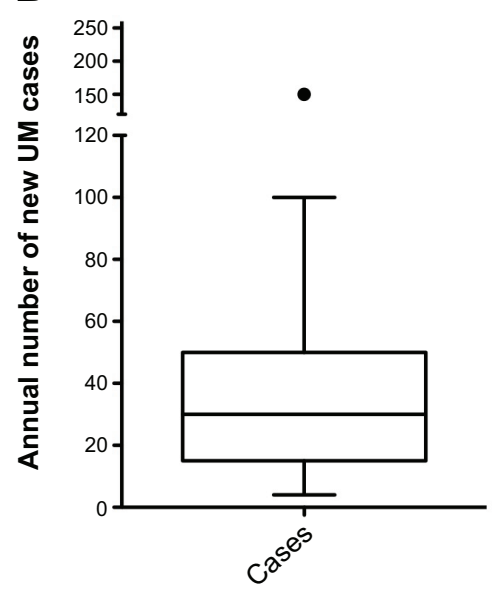

Figure 2 Number of new UM cases and biopsy activity per responding physician, collected from the clinician surveys in 2012 (number of participating physicians ( $\mathrm{n}$ ) =50) and early 2014 (number of participating physicians $(n)=35$ ).

Notes: Box plots with Tukey analysis. (A) Data derived from the 2012 survey: number of new UM cases per reporting physician (median: 35 , mean: 56 ) and annual number of biopsies (median: 15, mean: 27). (B) Data derived from the 2014 survey: number of new UM cases per reporting physician (median: 30, mean: 40).

Abbreviation: UM, uveal melanoma. 
better results in general whenever a transscleral approach was possible. Very posterior tumors were more likely to be approached transvitreally. There was almost an even split between biopsy procedures performed via a scleral window (57\%) or via a full thickness sclera (43\%). Approximately half of respondents $(54 \%)$ sealed the biopsy site, while the other half did not. FNAB specimens were usually preserved in the fixative kit provided by the testing lab (83\%).

In the 2014 survey, when patients were amenable to radiotherapy, $94 \%$ of respondents used brachytherapy primarily or exclusively (32 out of 34 ) and two used proton beam therapy. Patients undergoing radiotherapy were offered some type of molecular analysis of their pre-radiation tumor biopsy tissue "nearly always" by $76 \%$ of respondents $(\mathrm{N}=35)$ (Figure $3 \mathrm{~A})$. Four of the five respondents who "never or rarely" offered molecular analysis cited safety concerns. Three stated that the information would not alter their patients' management, and three respondents gave other reasons. One perceived the biopsy procedure as technically difficult. One believed this type of analysis should only be used in an investigational setting, not in routine clinical practice. The other respondent did not believe the information would be useful to the patient.

Similar to the 2012 survey, a minority of UM patients (median: 12.5\%; mean: 22\% $\pm 5 \%$ ) in the 2014 survey were considered ineligible for a tumor biopsy procedure, due to safety concerns associated with the surgery, such as small tumor size or tumor location $(\mathrm{N}=32)$. Of the remaining radiotherapy patients, for whom there was no biopsy safety concern and for whom cytogenetic or GEP analyses were offered, a median of $90 \%$ agreed to testing (mean: $69 \% \pm 6 \%$ ). The primary reasons for declining the tests were that the patient did not want to know $(65 \%$; $N=26)$, safety concerns about the

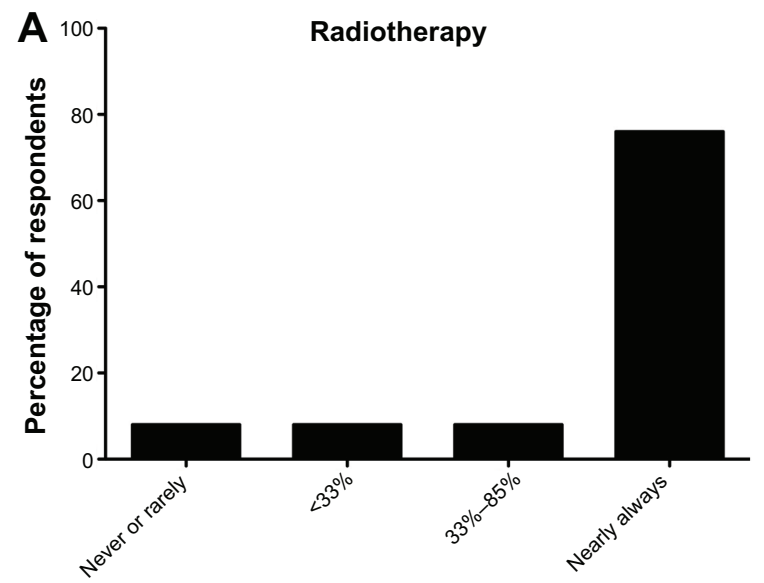

procedure (23\%), the cost of the testing (two respondents), or concerns about discrimination against them by insurance companies (one respondent).

\section{Surveys of clinicians: tumor biopsy analyses}

The majority of respondents (77\%) in the 2012 survey offered all patients a biopsy and some form of molecular tumor analysis. Some of the reasons for not offering a biopsy and testing included: 1) the tumor being too small and close to the macula, 2) the lack of treatments proven to improve clinical outcomes, 3 ) vision loss outweighing any potential benefits, and 4) lack of coverage for the procedures by some medical insurance carriers. FNAB specimens were analyzed by cytology (49\%), chromosomal analysis $(20 \%)$, and/or GEP $(89 \%)$ (Figure 4). Six percent were used only for research purposes. Fourteen percent were preserved in a tissue bank or used for a combination of research and other tests. Among respondents to the 2012 survey, 19\% offered CHR3 to most of their UM patients; $13.5 \%$ offered CHR3 to all patients, and the majority (65\%) offered CHR3 to none of their patients (Figure 5A). Seventy-eight percent of respondents offered molecular testing using GEP analysis to most of their UM patients; $54.1 \%$ offered GEP analysis to all patients, and $8 \%$ offered GEP analysis to none of their patients. The 2014 survey suggested a shift towards more respondents offering all their patients GEP and none of their patients CHR3 (Figure 5B). The median percentage of patients in the 2012 survey who declined to have their tumor analyzed at all was $25 \%$ (mean $38 \% \pm 6 \%$ ). Elderly patients were generally not interested, some patients feared loss of their medical insurance if a definitive diagnosis was added to their medical

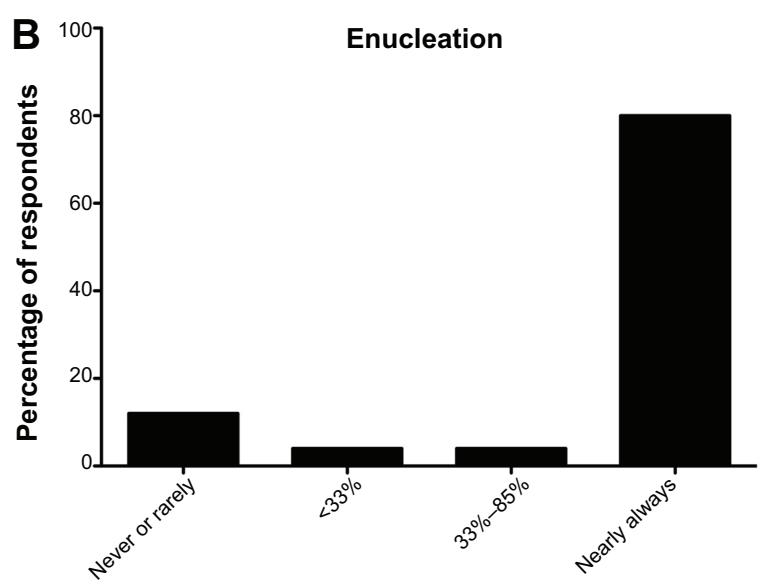

Figure 3 Distribution of physicians according to the frequency with which they offer some type of tumor analysis (CHR3 or GEP analysis) by (A) patients undergoing radiotherapy, or $(\mathbf{B})$ patients undergoing enucleation.

Notes: Data derived from the 2014 survey. $\mathrm{N}=35$.

Abbreviations: UM, uveal melanoma; CHR3, chromosome 3 analysis; GEP, gene expression profiling. 


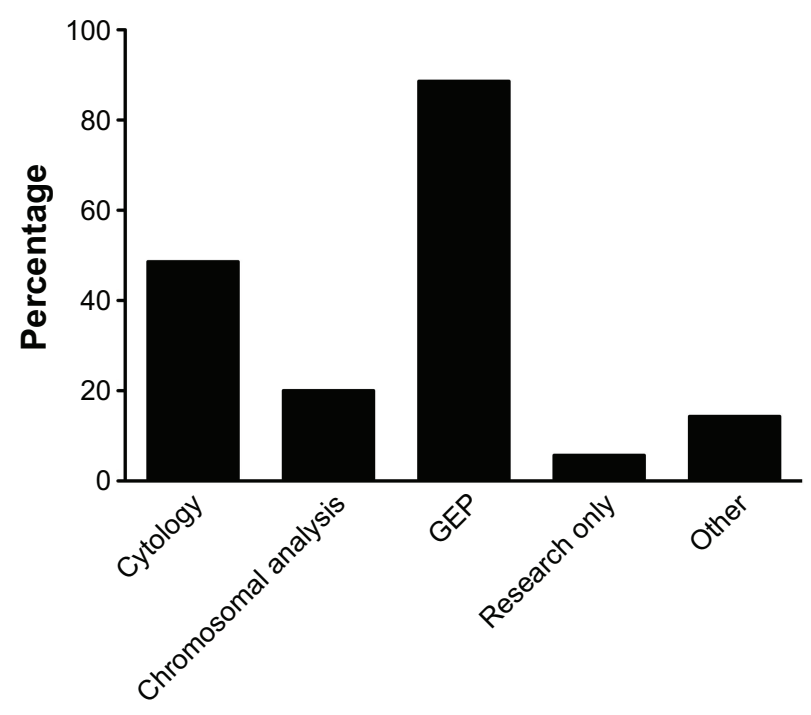

Figure 4 Test services performed on biopsy specimens.

Notes: The majority of clinicians offered UM patients a biopsy and some form of molecular tumor analysis. $\mathrm{N}=35$.

Abbreviations: GEP, gene expression profiling; UM, uveal melanoma.

records, and some feared the biopsy procedure would hasten tumor metastasis or cause additional morbidities. The general absence of proven treatment interventions was also a patient reason for declining biopsy and testing.

In the 2014 study, patients undergoing enucleation for UM were offered some type of cytogenetic (CHR3) and/ or molecular profiling analysis (GEP) of the tumor by $83 \%$ of the respondents (Figure 3B). Reasons given by the four respondents who never offered these analyses were: 1) the perceived technical difficulty of the biopsy procedure, 2) did not believe the information would be useful to the patients, and 3) did not believe the information would be useful to the respondent. The option of having the testing done after referral to a surgeon was discussed with UM patients by three respondents who did not offer the testing through their own practice.

\section{Effects on treatment strategy}

Perhaps most importantly, 74\% of respondents in the 2012 survey used the information obtained from the FNAB specimens and cytogenetic or GEP analyses to change the frequency of metastatic disease surveillance (Figure 6). Twenty-one percent ( 8 out of 39) did not use the information in the management of their patients, and two of these respondents referred their UM patients to oncologists, who did use the genetic information. Four respondents who did not use the cytogenetic data cited the current lack of effective therapeutic options. For patients at higher risk of metastasis, $15 \%$ also offered prophylactic therapy and $23 \%$ offered participation in a clinical trial of an investigational therapy.

Similarly, $79 \%$ of respondents in the 2014 survey ( $=33$ ) used the information obtained from the cytogenetic or GEP analyses to change their clinical practice, such as adjusting the frequency of metastatic disease surveillance, referral to medical oncology for follow-up, and/or counseling/referral regarding adjuvant treatment or clinical trials. Patients with low metastatic risk, based on CHR3 or a Class 1 GEP result, were generally assigned to less frequent (every 6 or 12 months) and less intensive clinical visits. Most respondents performed liver function tests and some type of hepatic imaging (eg, CT, ultrasound, MRI) at least once a year in low-risk UM patients. Only one respondent counseled or referred low-risk patients for adjuvant clinical trials or adjuvant treatment. In contrast, patients with high metastatic risk, based on CHR3 or a Class 2 GEP result, were assigned
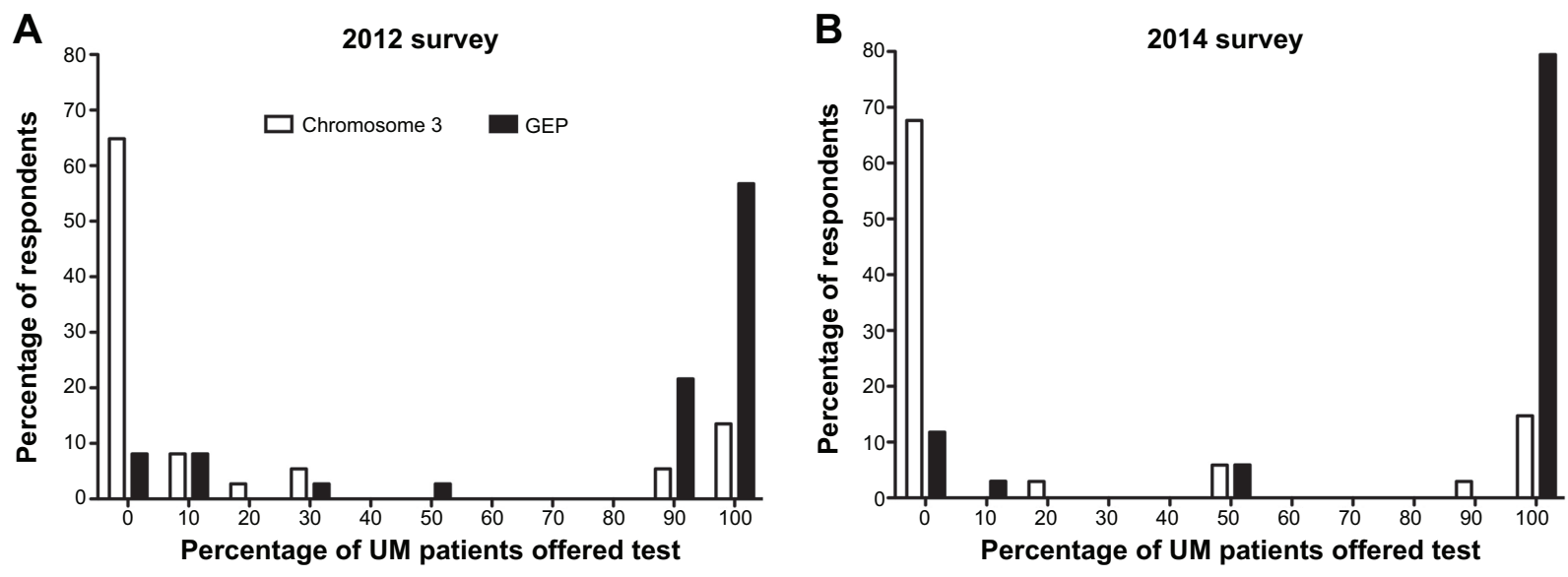

Figure 5 Histograms illustrating the distribution of UM patients offered each type of diagnostic test.

Notes: (A) Data derived from the 2012 survey; $N=37$. (B) Data derived from the 2014 survey; $N=34$

Abbreviations: UM, uveal melanoma; GEP, gene expression profiling. 


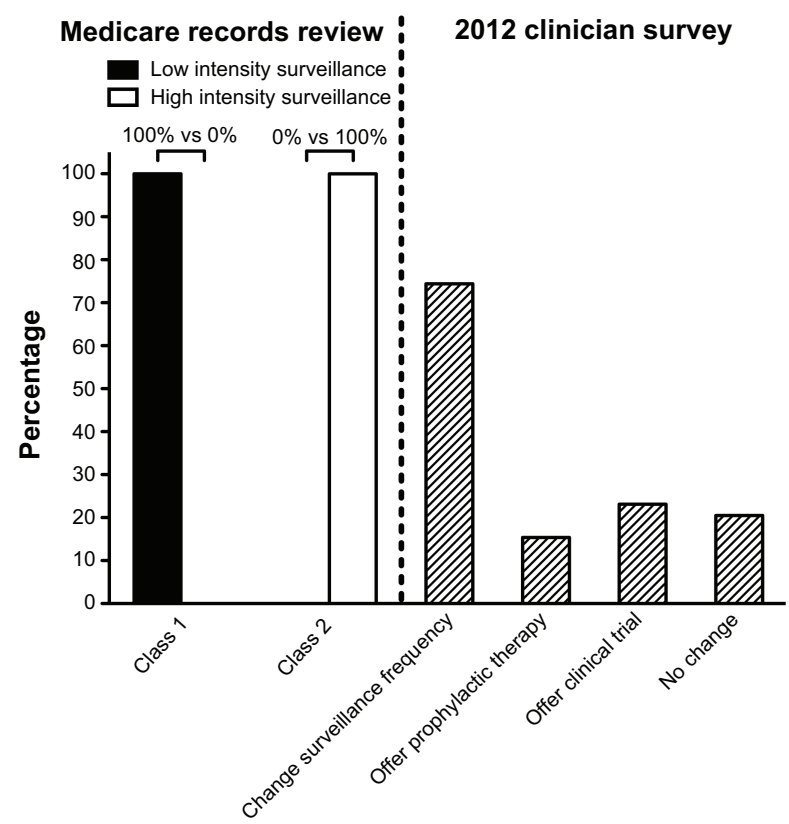

Figure 6 Clinical use of test data for patient management.

Notes: High-intensity surveillance of UM patients was defined as clinical visits every 3-6 months, liver function tests every 3-6 months, and liver imaging/systemic evaluation (eg, CT, ultrasound, MRI) every 3-6 months. Low-intensity surveillance of UM patients was defined as clinical visits every 6-12 months and liver function tests with some type of hepatic imaging at least once a year. Records review: $\mathrm{N}=\mathbf{8 8}$. Survey: $\mathrm{N}=39$.

Abbreviations: UM, uveal melanoma; CT, computed tomography; MRI, magnetic resonance imaging.

to more frequent surveillance, with hepatic imaging or liver function testing, often alternating quarterly. If the diagnosing physician was not an oncology specialist, then high-risk patients were referred to one. Only one respondent said they did not counsel or refer high-risk patients for adjuvant clinical trials or adjuvant treatment.

When asked their thoughts concerning a low-morbidity, low-toxicity clinical trial in the future that was designed as an adjuvant treatment protocol, 38\% believed the protocol should be offered to all UM patients and 59\% believed the protocol should be offered only to UM patients at high risk of tumor metastasis, based on cytogenetic or GEP analyses $(\mathrm{N}=34)$. Five of the respondents $(15 \%)$ who believed only high-risk patients should be offered this type of clinical trial would also refer patients who were classified as high-risk based on clinical findings (ie, tumor size, cell type, etc) to this type of clinical trial. None of the respondents would fail to offer this alternative to their high-risk patients.

Respondents were more cautious about referring UM patients to a high-morbidity, high-toxicity clinical trial that was designed as an adjuvant treatment protocol $(\mathrm{N}=34)$. Only $9 \%$ agreed that it should be offered to all UM patients. The majority (74\%) believe that patients classified as high-risk, based on cytogenetic or GEP analyses, should be offered this alternative. Six of these respondents would also offer it to UM patients classified as high-risk based on clinical findings, and an additional two respondents would offer this type of clinical trial to UM patients classified as high-risk based only on clinical findings. There were also four respondents $(12 \%)$ who would not offer this type of clinical trial to any UM patient.

Of the 191 evaluable patients in the Medicare records review, there were 88 (46\%) with documented medical treatment actions in their medical records or with documented institutional medical policies related to surveillance plans (Figure 1). All of the GEP Class 1 UM patients were treated with a low-intensity surveillance plan (Figure 6). In contrast, all of the GEP Class 2 UM patients were treated with a high-intensity surveillance plan $(P<0.0001$ versus Class 1 surveillance). Comments on GEP Class 1 surveillance practices from patient medical charts indicated that physicians advised liver function tests on an annual or biannual basis, liver imaging on an annual basis, and an annual systemic evaluation or no surveillance at all, due to the low risk of UM tumor metastasis. In contrast, GEP Class 2 surveillance practices from patient medical charts indicated that physicians advised liver function tests every 3, 4, or 6 months, and liver imaging/systemic evaluation every 3 to 6 months. The majority of records indicated a "known policy" of more frequent and intensive surveillance for Class 2 patients. Of the 191 evaluable patients, there were 36 (19\%) with information concerning referrals to medical oncology after initial diagnosis. All 23 Class 2 patients were referred to medical oncology, but none of the 13 Class 1 patients $(P<0.0001$ versus Class 1). Similarly, only Class 2 patients were recommended for adjunctive treatment regimens.

\section{Discussion}

Concomitant with the completion of the GEP test prospective, multicenter clinical validation study, molecular testing for analyzing uveal melanomas has become more easily accessible over the past few years. However, the frequency of use of these tests by ocular oncologists and the clinical application of these analyses have not been previously assessed. Our surveys found a number of interesting observations about current clinical practices.

In the first survey the physician respondents practiced in North America, South America, and Europe; although, it should be noted that a commercial GEP test is not currently available in Europe or South America. Therefore, the 2014 survey only queried physicians with clinical practices in the United States. In this latest survey, the respondents were 
evenly split between university-based and private practices, and most were the only physician in their practice group to treat UM patients. Overall, there was a wide range in the UM caseloads among the different clinical practices. Most handled 30-35 new cases a year, but a few had 100 or more, for an estimated total of 1,389 in the 2014 survey. Based on the US National Cancer Institute estimate of 4.3 new cases of UM per million US adults a year, the most recent survey may represent all or nearly all of the annual UM caseload in the US in $2013 .{ }^{19,20}$ In both survey groups, more than $75 \%$ of respondents offered molecular testing of tumor biopsy samples to most or all of their UM patients. Safety concerns rendered a median $10 \%$ of UM patients ineligible for a biopsy procedure. Of the remaining patients, for whom there was no biopsy safety concern and for whom CHR3 or GEP were offered, the majority agreed to testing. The primary reasons patients declined testing were that they did not want to know, they had safety concerns about the biopsy procedure, or they had concerns about discrimination against them by insurance companies. Elderly patients were generally less interested.

The physicians preferred a 25 or 27 gauge needle for FNAB of UM tumors, generally attached to a $10 \mathrm{cc}$ syringe via flexible tubing. The choice of surgical approach was usually determined by tumor location and depth, although several respondents indicated better results in general whenever a transscleral approach was possible. Very posterior tumors were more likely to be approached transvitreally. There was almost an even split between biopsy procedures performed via a scleral window or via a full thickness sclera. When the tumors were amenable to radiotherapy, the vast majority were treated using brachytherapy.

As might be expected with the widespread adoption of a new technology, the majority of physicians used the information obtained from molecular analyses to change patient management, specifically the frequency of metastatic disease surveillance. The ability to rule out high-risk disease represents an important shift in UM patient management. According to the survey data, patients at high risk for tumor metastases were monitored every 3-6 months, compared with every 6-12 months for low-risk patients. The risk categories generated from this type of testing were also used to identify UM patients (high-risk) most likely to be referred to, and most likely to benefit from, investigational clinical trials.

The Medicare chart and policy review results parallel the results from the two blinded clinician surveys. Specifically, of cases for which there was documented evidence of clinical use of the results and/or known physician policy, 100\% of patients with a low-risk Class 1 result were managed by a low-intensity surveillance plan that was primarily defined as liver function tests without (or with) abdominal ultrasound at a frequency of one or two times per year. In contrast, 100\% of patients with a high-risk Class 2 result were managed by a high-intensity surveillance plan, primarily defined as liver function tests and abdominal ultrasound, CT, and/or positron emission tomography (PET) at a frequency of 2 to 4 times per year. Additionally, more Class 2 patients received referrals to medical oncology and/or adjuvant treatment protocols.

In agreement with the results obtained in the clinician surveys and the Medicare chart and policy study, molecular diagnostic testing (specifically GEP) was shown to have a significant impact on clinical treatment strategy. ${ }^{4}$ Clinicians used these data to "rule out" high-risk disease. When GEP testing was used, nearly all patients with GEP Class 1 UM were managed with a low-intensity surveillance paradigm. In contrast, almost all Class 2 UM patients received the standard high-intensity surveillance. Consistent with their higher-risk disease, Class 2 UM patients were more likely to be referred to a medical oncologist for possible clinical trial enrollment. Thus, the GEP results may enable low-risk patients to avoid unnecessary monitoring, inconvenience and cost, and the adverse personal effects of worrying about their disease. These results also provided high-risk patients and their caregivers with important information for making informed decisions about their own health care. Recently, Correa and Augsburger ${ }^{21}$ reported data showing that 29\% of Class 2 GEP UM patients, but only $6 \%$ of Class 1 GEP patients, had tumor metastases at a median follow-up time of 32.5 months (total sample size: 158). Also, GEP classification was "substantially better" than cytologic classification for predicting metastasis and metastatic death. Together, these data support a recommendation for more intensive surveillance in Class 2 GEP patients, which will hopefully one day translate into better mortality outcomes.

Overall, the data in this report support the conclusion that molecular analysis, including GEP and chromosomal analysis, have been widely accepted and adopted for uveal melanoma treatment decisions. In addition to the impact on surveillance and referral management, such information is likely to be required for entry into future clinical trials involving adjuvant therapy at major medical centers. ${ }^{22}$ The authors recognize that there is no strong data suggesting that more intensive surveillance improves survival outcomes. However, the recent data showing benefit using immunotherapy ${ }^{23}$ and targeted therapy ${ }^{24}$ is encouraging in that these clinical trials may advance immediate, adjuvant therapy options for UM patients over the next few years and ultimately reduce mortality rates. 


\section{Acknowledgments}

The authors thank Loretta L Nielsen, PhD (LLNielsen Medical Writing LLC, Ramona, CA) for medical writing services; John Maetzold, Clare Johnson, RN, and Trisha Sappington for data extraction services and administrative support.

Financial support was received from Castle Biosciences, Inc. (Friendswood, TX, USA). The sponsor participated in study design, conducting the study, data collection, data management, data analysis, interpretation of the data, and the preparation and review of the manuscript.

\section{Disclosure}

The authors report no conflicts of interest in this work.

\section{References}

1. Singh AD, Topham A. Incidence of uveal melanoma in the United States: 1973-1997. Ophthalmology. 2003;110(5):956-961.

2. Kujala E, Makitie T, Kivela T. Very long-term prognosis of patients with malignant uveal melanoma. Invest Ophthalmol Vis Sci. 2003;44(11): 4651-4659.

3. Harbour JW. A prognostic test to predict the risk of metastasis in uveal melanoma based on a 15-gene expression profile. Methods Mol Biol. 2014;1102:427-440.

4. Harbour JW, Chen R. The DecisionDx-UM gene expression profile test provides risk stratification and individualized patient care in uveal melanoma. PLOS Curr. 2013;5. doi:10.1371/currents.eogt. af8ba80fc776c8f1ce8f5dc485d4a618.

5. Collaborative Ocular Melanoma Study Group. Assessment of metastatic disease status at death in 435 patients with large choroidal melanoma in the Collaborative Ocular Melanoma study (COMS). COMS report no. 15. Arch Ophthalmol. 2001;119(5):670-676.

6. American Joint Committee on Cancer (AJCC). [homepage on the Internet]. Chicago: AJCC; [cited 2014 Jan 16]. Available from: https:// cancerstaging.org/Pages/default.aspx

7. National Comprehensive Cancer Network ( $\mathrm{NCCN})$. [homepage on the Internet]. Fort Washington, PA: NCCN; [cited 2014 Jan 16]. Available from: http://www.ncen.org/default.aspx

8. Pereira PR, Odashiro AN, Lim LA, et al. Current and emerging treatment options for uveal melanoma. Clin Ophthalmol. 2013;7:1669-1682.

9. Bronkhorst IHG, Maat W, Jordanova ES, et al. Effect of heterogeneous distribution of monosomy 3 on prognosis in uveal melanoma. Arch Pathol Lab Med. 2011;135(8):1042-1047.

10. Maat W, Jordanova ES, van Zelderen-Bhola SL, et al. The heterogeneous distribution of monosomy 3 in uveal melanomas: implications for prognostication based on fine-needle aspiration biopsies. Arch Pathol Lab Med. 2007;131(1):91-96.
11. Schoenfield L, Pettay J, Tubbs RR, Singh AD. Variation of monosomy 3 status within uveal melanoma. Arch Pathol Lab Med. 2009;133(8): 1219-1222.

12. Mensink HW, Vaarwater J, Kilic E, et al. Chromosome 3 intratumor heterogeneity in uveal melanoma. Invest Ophthalmol Vis Sci. 2009;50(2): 500-504.

13. Midena E, Bonaldi L, Parrozzani R, Tebaldi E, Boccassini B, Vujosevic S. In vivo detection of monosomy 3 in eyes with mediumsized uveal melanoma using transscleral fine needle aspiration biopsy. Eur J Ophthalmol. 2006;16(3):422-425.

14. Young TA, Rao NP, Glasgow BJ, Moral JN, Straatsma BR. Fluorescent in situ hybridization for monosomy 3 via 30 -gauge fine-needle aspiration biopsy of choroidal melanoma in vivo. Ophthalmology. 2007;114(1):142-146.

15. Desjardins L, Lumbroso-Le Rouic L, Levy-Gabriel C, et al. FNA biopsies for genomic analysis and adjuvant therapy for uveal melanoma. Acta Ophthalmol. 2010;88(s246).

16. Onken MD, Worley LA, Char DH, et al. Collaborative Ocular Oncology Group report number 1: prospective validation of a multi-gene prognostic assay in uveal melanoma. Ophthalmology. 2012;119(8): 1596-1603.

17. Onken MD, Worley LA, Harbour JW. Association between gene expression profile, proliferation and metastasis in uveal melanoma. Curr Eye Res. 2010;35(9):857-863.

18. American Joint Committee on Cancer. AJCC Cancer Staging Manual, 7th edition. Philadelphia: Springer; 2010. Malignant melanoma of the uvea, p. 547-553.

19. US National Cancer Institute at the National Institutes of Health General information about intraocular (uveal) melanoma treatment. [cited $2014 \mathrm{Feb} 18]$. Available from: http://www.cancer.gov/ cancertopics/pdq/treatment/intraocularmelanoma/HealthProfessional/ page 1

20. US Census Bureau. Census Bureau projects US population of 317.3 million on New Year's Day. [cited 2014 Feb 18]. Available from: http://www.census.gov/newsroom/releases/archives/population/cb13tps112.html

21. Correa ZM, Augsburger JJ. Sufficiency of FNAB aspirates of posterior uveal melanoma for cytologic versus GEP classification in 159 patients, and relative prognostic significance of these classifications. Graefes Arch Clin Exp Ophthalmol. 2014;252(1):131-135.

22. US National Institutes of Health. ClinicalTrials.gov. Uveal melanoma clinical trials database. [cited 2014 Jan 16]. Available from: http://www. clinicaltrials.gov

23. Luke JJ, Callahan MK, Postow MA, et al. Clinical activity of ipilimumab for metastatic uveal melanoma: a retrospective review of the DanaFarber Cancer Institute, Massachusetts General Hospital, Memorial Sloan-Kettering Cancer Center, and University Hospital of Lausanne experience. Cancer. 2013;119(20):3687-3695.

24. Carvajal RD, Sosman JA, Quevedo F, et al. Phase II study of selumetinib (sel) versus temozolomide (TMZ) in gnaq/Gna11 (Gq/11) mutant (mut) uveal melanoma (UM). J Clin Oncol. 2013;31(15): Abstr CRA9003. 


\section{Supplementary materials}

Table SI 2012 survey questions

I How many new cases of uveal melanoma do you see annually? (Respondent $\mathrm{N}=50$ )

2 Do you perform some type of cellular and/or molecular analysis of uveal malignant melanoma which requires a fine needle biopsy? (Respondent $\mathrm{N}=50$ )

3 How many uveal melanoma patients (\%) are not eligible for biopsy due to safety concerns? (Respondent $\mathrm{N}=37$ )

4 For eligible patients do you perform analysis for: (Respondent $\mathrm{N}=37$ )

a. Enucleated eyes only

b. All cases

5 What percent of uveal melanoma cases do you offer cytogenetic (chromosome 3) testing? (Respondent $\mathrm{N}=37$ )

6 What percent of uveal melanoma cases do you offer molecular testing (DecisionDx-UM gene expression profile)? (Respondent $\mathrm{N}=37$ )

7 How many biopsies for uveal melanoma do you perform annually? (Respondent $\mathrm{N}=37$ )

8 In what \% of cases do you employ use of a vitrector for biopsy procedures? (Respondent $\mathrm{N}=35$ )

9 What size needle gauge do you use to biopsy the tumor? (Respondent $\mathrm{N}=35$ )

10 Do you utilize flexible tubing between the needle hub and the syringe to stabilize the needle position? (Respondent $\mathrm{N}=27$ )

II What size syringe do you use in order to create vacuum? (Respondent $\mathrm{N}=27$ )

12 Do you perform the biopsy via a scleral window or via full thickness sclera? (Respondent $\mathrm{N}=35$ )

13 What percent of biopsies are performed transscleral? (Respondent $\mathrm{N}=35$ )

14 What percent of biopsies are performed transvitreal? (Respondent $\mathrm{N}=35$ )

15 If you perform both transscleral and transvitreal biopsies, what factors contribute to your decision process? (Respondent $\mathrm{N}=35$ )

16 After the biopsy is performed do you "seal" the biopsy site? (Respondent $\mathrm{N}=35$ )

17 Is the biopsy performed... (Respondent $\mathrm{N}=35$ )

a. At the time of plaque placement, enucleation or clip placement

b. As a separate procedure

18 How many tumor sites do you biopsy? (Respondent $\mathrm{N}=24$ )

19 What testing do you perform with your biopsy material? (Respondent $\mathrm{N}=35$ )

20 What fixative do you use (ex, fixative provided by processing lab, cytolyte, formalin)? (Respondent $\mathrm{N}=35$ )

21 Do you offer all patients biopsy and analysis of the tumor? (Respondent $\mathrm{N}=39$ )

22 What percentage of patients decline the offer for testing? (Respondent $\mathrm{N}=39$ )

23 What reasons are given for declining testing? (Respondent $\mathrm{N}=39$ )

24 How do you use the information clinically? (Respondent $\mathrm{N}=39$ ) 
Table S2 2014 survey questions

I Did you participate in the July 2012 survey that Dr Aaberg distributed in preparation for the 2012 Retina sub-specialty day presentation? (Respondent $\mathrm{N}=35$ )

2 Are you: (Respondent $\mathrm{N}=32$ )

- University based

- Private practice

- Government based (ex: Veterans Administration)

- Other

3 Within your practice group, how many physicians treat primary uveal melanoma? (Respondent $\mathrm{N}=35$ )

4 How many new cases of uveal melanoma do you personally see annually (eg, if in a practice group with more than one physician who treats uveal melanoma, only include your direct new cases)? (Respondent $\mathrm{N}=35$ )

5 For patients amenable to radiotherapy, do you use: (Respondent $\mathrm{N}=34$ )

- Brachytherapy

- Proton Beam

- I have access to both modalities and use both equally

- I have access to both modalities and use Proton Beam principally

- I have access to both modalities and use Brachytherapy principally

- Other

6 For patients undergoing radiotherapy for ocular melanoma, do you offer some type of cytogenetic (chromosome 3) and/or molecular profiling analysis (the gene expression profile test) of the tumor which requires a biopsy? (Respondent $\mathrm{N}=35$ )

- Never or rarely

- Less than $33 \%$

- $33 \%-85 \%$

- Nearly always

7 If you answered "Never or rarely" to question \#5, you do not offer testing because (please check all answers that apply): (Respondent N=5)

- Safety concerns, such as increased risk of orbital seeding or risk of vision loss due to hemorrhage

- Perceive the biopsy procedure as technically difficult

- The information obtained will not alter my management decisions

- Do not believe the information provided to be useful to me

- Do not believe the information provided to be useful to the patient

- Believe the testing should only be used in an investigational setting

- Other

8 For patients undergoing enucleation for ocular melanoma, do you offer some type of cytogenetic (chromosome 3 - disomy or monosomy) and/ or molecular profiling analysis (the gene expression profile test - Class I or Class 2) of the tumor? (Respondent $\mathrm{N}=35$ )

- Never or rarely

- Less than $33 \%$

- $33 \%-85 \%$

- Nearly always

9 If you answered "Never or rarely" to question \#7 you do not offer testing because (please check all answers that apply): (Respondent $\mathrm{N}=2$ )

- Safety concerns, such as increased risk of orbital seeding or risk of vision loss due to hemorrhage

- Perceive the biopsy procedure as technically difficult

- The information obtained will not alter my management decisions

- Do not believe the information provided to be useful to me

- Do not believe the information provided to be useful to the patient

- Believe the testing should only be used in an investigational setting

- Other

I0 If you do not offer any form of testing in any case, do you still discuss the option with the patient and refer the patient to a center that does perform testing? (Respondent $\mathrm{N}=3 \mathrm{I}$ )

- Yes

- No

- Does not apply, because I do offer testing

II If you do not offer any form of testing in any case, please complete this question but you do not have to continue the questionnaire. Will you consider performing testing in the future? If so, what would have to occur? (Respondent $\mathrm{N}=26$ )

- No

- Yes,

- Does not apply, because I do offer testing

(Continued) 


\section{Table S2 (Continued)}

12 What percentage of your radiotherapy patients are not eligible for biopsy due to safety concerns related to the biopsy procedure, such as tumor location or small size? (Respondent $\mathrm{N}=32$ )

13 Of the remaining radiotherapy patients for whom there is no biopsy safety concern and for whom you offer cytogenetic or gene expression profiling analysis of the tumor, what percentage agrees to testing? (Respondent $\mathrm{N}=32$ )

14 What is the principle reason that patients decline testing (select only what you consider the number one reason even though other reasons may apply)? (Respondent $\mathrm{N}=26$ )

- Safety concerns, for example: increased risk of orbital seeding, risk of vision loss due to hemorrhage.

- The patient "does not want to know"

- Concerned that the information may be used against them, example: disability or life insurance company may deem the patients as "uninsurable" if they have a "high-risk" tumor.

- Cost of testing

- Other

15 Which testing do you offer? (check all that apply) (Respondent $\mathrm{N}=34$ )

- Cytogenetics (chromosome 3 analysis)

- Molecular profiling diagnostics (the gene expression profile test)

- Other

16 For those patients that have testing, what percent do you offer cytogenetic (chromosome 3) testing? (Respondent $\mathrm{N}=32$ )

17 For those patients that have testing, what percent do you offer molecular profiling diagnostics (the gene expression profile test)? (Respondent $\mathrm{N}=34$ )

18 For those patients who have testing performed, do you use the information clinically (such as metastatic surveillance approach, referral to medical oncology for follow-up, or counsel/refer regarding adjuvant treatment or adjuvant clinical trials)? (Respondent $\mathrm{N}=33$ )

19 If you answered "Yes" to question 18 regarding metastatic surveillance approach, please describe your general approach (eg, frequency and type of testing or imaging) for patients with low risk Class I/disomy 3 test results. (Respondent $\mathrm{N}=24$ )

20 If you answered "Yes" to question 18 regarding metastatic surveillance approach, please describe your general approach (eg, frequency and type of testing or imaging) for patients with high-risk Class 2 /monosomy 3 test results. (Respondent $N=24$ )

21 If you answered "Yes" to question 18 regarding counseling regarding adjuvant treatment options or adjuvant clinical trials (Respondent $\mathrm{N}=27$ ):

- Do you counsel or refer low risk Class I/disomy 3 patients for adjuvant clinical trials or adjuvant treatment?

- Do you counsel or refer high-risk Class 2 /monosomy 3 patients for adjuvant clinical trials or adjuvant treatment?

22 Thinking about future clinical trials, if a low morbidity risk adjuvant treatment protocol (that is, a treatment that has a relatively low toxicity or side effect profile) was available for uveal melanoma patients, do you believe (Respondent $\mathrm{N}=34$ ):

- The protocol should be offered to all uveal melanoma patients

- The protocol should be offered only to patients with high-risk features based on clinical findings (ie, tumor size, cell type, etc).

- The protocol should be offered only to patients with high-risk features based on gene expression profile diagnostics and/or cytogenetics.

- The protocol should not be offered to any patient.

23 Thinking about future clinical trials, if a high morbidity risk adjuvant treatment protocol (that is, a treatment that has a relatively high toxicity or side effect profile) was available for uveal melanoma patients, do you believe (Respondent $\mathrm{N}=34$ ):

- The protocol should be offered to all uveal melanoma patients

- The protocol should be offered only to patients with high-risk features based on clinical findings (ie, tumor size, cell type, etc).

- The protocol should be offered only to patients with high-risk features based on molecular diagnostics and/or cytogenetics.

- The protocol should not be offered to any patient.

Clinical Ophthalmology

\section{Publish your work in this journal}

Clinical Ophthalmology is an international, peer-reviewed journal covering all subspecialties within ophthalmology. Key topics include: Optometry; Visual science; Pharmacology and drug therapy in eye diseases; Basic Sciences; Primary and Secondary eye care; Patient Safety and Quality of Care Improvements. This journal is indexed on

\section{Dovepress}

PubMed Central and CAS, and is the official journal of The Society of Clinical Ophthalmology (SCO). The manuscript management system is completely online and includes a very quick and fair peer-review system, which is all easy to use. Visit http://www.dovepress.com/ testimonials.php to read real quotes from published authors. 